CATÁSTROFE Y SUBJETIVIDAD POLÍTICA

Pilar Errázuriz Vidal 


\section{PILAR ERRÁZURIZ}

Psicoterapeuta, Psicoanalista. Doctora en Estudios de Género, Universidad de Valladolid. Magíster en Psicología, Universidad de la Sorbonne, París. Directora del Centro de Género y Cultura en América Latina de la Facultad de Filosofía y Humanidades de la Universidad de Chile. Profesora Asociada a cargo del curso de Psicoanálisis y Género en el Magíster de Género de la Universidad de Chile. Miembro activo de la Asociación Internacional de Psicoterapia de Grupo. 
EN SITUACIÓN CATASTRÓFICA al menos dos fenómenos emergen en un estudio diagnóstico psicosocial de una comunidad damnificada: la calidad del tejido social y el grado de subjetividad política de los/las ciudadanos/as. A mayor cohesión vincular y mayor conciencia subjetiva de su inscripción comunitaria, mayor y mejor capacidad de resiliencia.

Para una efectiva intervención psicosocial en situación de crisis colectiva es necesario, entonces, establecer un primer diagnóstico no solo del material consciente, explícito y manifiesto que vehicula todo relato, sino del discurso implícito y latente que permita interpretar el grado de consistencia vincular intersubjetiva y comunitaria. Con ese fin, la intervención diagnóstica psicosocial dinámica dispone de varios instrumentos al servicio de las técnicas de entrevistas individuales y de trabajo en Grupo Operativo ${ }^{1}$. Los instrumentos que pueden utilizar los/las psicólogos/as formados en teoría psicoanalítica individual y grupal son precisos: la pregunta, el señalamiento, la construcción y, finalmente, la interpretación del material latente/inconsciente.

Sobre la base de la epistemología feminista, entendemos que la vinculación que establecen los psicólogos con una población dañada, genera una instancia asimétrica inevitable y que todas las intervenciones terapéuticas obedecen al stand point del profesional o investigador. De manera que, si bien para la población afectada la presencia de profesionales de la salud en el lugar de la catástrofe cuenta a priori con una expectativa conscientemente receptora, no siempre la transferencia que se establece va a despejar las resistencias o la hostilidad que provoca la propia relación "de poder" establecida, en particular cuando se trata de grupos socioeconómicamente vulnerables y vulnerados.

Asimismo, hay que asumir que toda interpretación de un discurso latente y/o inconsciente -niveles más profundos que lo simplemente implícito- solo llega hasta donde la sensibilidad y la formación del o la profesional alcanza.

1. Grupo Operativo, técnica desarrollada por la escuela de E. Pichón Riviére, la que a diferencia de los Grupos Focales o de Discusión, rastrean los niveles de las vinculaciones inconscientes, de las proyecciones, de las ansiedades y miedos existentes según los tres núcleos de la formación de la personalidad según estudios psicoanalíticos clásicos. 
Precisamente por estos recaudos, estas intervenciones se acompañan de un ejercicio representacional protagónico para los y las sujetos de la intervención, con perspectiva de género, lo que ayuda a consolidar las pertenencias grupales, institucionales y comunitarias que conforman su eje narcisista. Se trata de ejercitar con los y las participantes lo que hemos denominado gimnasia topológica y gimnasia cronológica ${ }^{2}$, que consiste en convocar al aquí y ahora diferentes momentos históricos de sus vínculos y experiencias psicosociales, al tiempo que se apela a sus posiciones dentro de las genealogías (masculinas, femeninas y ambas) tanto familiares como sociales en términos intersubjetivos (relaciones individuales), grupales, colectivos, institucionales y comunitarios.

Con los instrumentos que hemos mencionado, se pretende contener a los sujetos para que inicien la tarea de reconstitución del tejido social del mundo interno y externo que los sostiene, y que se han visto convulsionados por la indefensión producida a raíz de una situación catastrófica. Todo sujeto recorre la cotidianidad con lo que denominamos la aldea interna ${ }^{3}$, esto es la presencia consciente, preconsciente e inconsciente de los vínculos que ha internalizado a lo largo de su recorrido vital. Nos referimos a las figuras de identificación, modelos del ideal del yo, censuradores superyoicos, así como la interiorización de los pares, los iguales y los diversos, que perviven con mayor o menor intensidad al interior del sujeto y con quienes se dialoga consciente o inconscientemente. En este segundo registro, resulta posible rastrear los personajes más representativos de la aldea interna en los sueños, en los síntomas, en los lapsus, entre otros, así como en el material latente del discurso explícito.

La razón por la cual una situación de crisis de índole catastrófica provoca en los sujetos una desorganización psíquica en la aldea interna, es que la indefensión resultante produce -por analogía- una regresión a los resabios originales de la constitución psíquica infantil, momento en el que los vínculos aún no adquirían una identidad y presencia sólida en la representación interna. Como consecuencia, se estimulan, así, los tres núcleos de base: confusional, paranoide y depresivo, generando miedos a la disolución, al ataque y a la pérdida, que construyen instantáneamente ansiedades (confusional, paranoide, depresiva) ${ }^{4}$. Estas se suceden emocionalmente una tras otra en una suerte de acelerado circuito,

2. Errázuriz, Pilar. Psicología Social y Género: espacio a salvo para mujeres, Santiago de Chile: Ed. De la Elipse, 2006.

3. "La psicología individual es al mismo tiempo y desde un principio psicología social" nos enseña Freud en el artículo de 1921, "Psicología de las masas y análisis del yo", en Obras Completas, Biblioteca Nueva, 1981, p. 2563.

4. Pampliega de Quiroga, Ana. Proceso de la constitución del mundo interno, Buenos Aires: Ed. Cinco, 1992. 
o se superponen, causando síntomas tales como insomnio, trastornos alimentarios, fantasías de muerte e, incluso, intentos de suicidio. El tejido interno se ve conmovido y si el entramado social es precario y la subjetividad política no encuentra cabida en las instituciones y en la comunidad, el sujeto arriesga un empobrecimiento libidinal que le impide investir energía afectiva en una reconstrucción post catástrofe, tanto material como psíquica.

En 2010, como es sabido, nuestro país sufrió un terremoto de tal envergadura que zonas enteras quedaron devastadas. Desde el Proyecto Anillo SOC 21 de Estudios Interdisciplinarios de Género de la facultad de Filosofía de la Universidad de Chile, dirigido por la Dra. Kemy Oyarzún y en colaboración con un grupo de psicólogos/as de otras universidades, nuestro equipo psicosocial intervino en la comuna de Lolol y Paredones pertenecientes a la Región de O'Higgins, en los tres meses que siguieron a la catástrofe. Bajo la coordinación de quien suscribe, un grupo de 6 psicólogos/as ${ }^{5}$ trabajaron con 42 mujeres y 10 hombres con el objetivo de evaluar la calidad del tejido social utilizando las técnicas ya mencionadas, que tienen como propósito reforzar la cohesión social interna/externa y la subjetividad política de los/las involucrados/as, para aumentar su capacidad de resiliencia ${ }^{6}$.

El perfil etario de las 42 mujeres que participaron en esta intervención es amplio: desde menos de 31 años hasta más de 61. De ellas se cuentan 11 analfabetas. Un tercio ha completado su educación básica y solo un 9\% la media. La mitad de ellas son jefas de hogar (solteras, viudas, separadas), y solo 9 de las más jóvenes y de más edad no tienen hijos. La situación laboral de todas ellas es precaria: recolectoras de restos de cosecha y frutos silvestres, algueras en la costa cercana a la comuna, temporeras de la vendimia y agricultoras por cuenta propia. Un grupo menor se dedica a labores del hogar.

De los 10 hombres que participaron de la intervención, un 50\% eran mayores de 61 años y no se integraron hombres menores de 31. El nivel educacional es inferior al de las mujeres, no se cuenta con ninguno que haya completado la educación media, solo un tercio terminó la básica, y un tercio es analfabeto. La carencia de educación es creciente según aumenta la edad de los varones. Todos ellos, menos 3, son casados. De los tres, uno es viudo y de los solteros, uno es el más joven y el segundo, el mayor de todos. Todos, menos los solteros, tienen hijos y son jefes de hogar. Al igual que en el grupo de mujeres, los trabajos de los

5. Maite Cortés Arteaga, Catalina Jara, Elisa Rojas, Paz Ulloa, Sebastián Morales y José María de Pablo, coordinados por Dra. Pilar Errázuriz Vidal.

6. La intervención e investigación que se realizó fue mucho más amplia, pero a efectos de este escrito solo nos referiremos a estos dos aspectos precisos: tejido social y subjetividad política, en el contexto del trabajo realizado por el equipo psicosocial clínico. 
varones son precarios: recolectores, temporeros, agricultores por cuenta propia y asalariados con escasa remuneración.

Luego de un riguroso catastro de la situación material de los damnificados realizado por el equipo de psicólogos/as, se procedió a un diagnóstico clínico de estas 52 personas damnificadas. Tanto en las mujeres como en los hombres, el diagnóstico clínico mostró cuadros de precariedad psicológica previa a la catástrofe, siendo esta última un agravante de las ansiedades y desencadenante de síntomas. Desde este primer diagnóstico se pudo observar un deterioro en el tejido social, mostrando una frágil cohesión colectiva, constituyéndose la comunidad en una serialidad de grupos familiares aislados e incomunicados entre sí, sin mayor empatía recíproca. Más tarde, tanto los contenidos de las entrevistas como el material que emergió en los Grupos Operativos mostraron los detalles y las implicaciones de este proceso de empobrecimiento del tejido comunitario como lo mostraremos más adelante.

Con este diagnóstico que indicaba un pronóstico dudoso por la recurrencia de las fantasías de muerte, intentos de suicidio, pérdida de las confianzas tanto en las instituciones como en la comunidad, procedimos a realizar 6 entrevistas psicológicas en profundidad a mujeres, según protocolo psicoanalítico de José Bleger, en las cuales participaron dos informantes clave.

Uno de los fenómenos que nos llamó la atención fue que las portavoces de la situación de la catástrofe y de la organización en situación de crisis y emergencia, fueron las mujeres mayores. Los familiares, juntos o dispersos, recurrieron a la abuela o madre de edad donde refugiarse, aún cuando los daños materiales afectaran a estas últimas de igual manera. Las mujeres mayores descritas como el referente más importante para hijas, yernos y nietos/as, constituyeron el aglutinador de los vínculos interpersonales. Asimismo, contrastando las intervenciones diagnósticas de hombres y mujeres, si bien ambos grupos se mostraron igualmente afectados psíquicamente por el terremoto, las mujeres eran quienes podían verbalizar con facilidad proyecciones de recuperación futura, mientras que los hombres mostraban un grado fatalista que se consolaba recordando mejores tiempos en el pasado.

Un segundo fenómeno que resultó común en el material recogido fue la toma de conciencia de la precariedad de los vínculos interpersonales en la comunidad y el deterioro paulatino que ha sufrido el tejido social por la transformación de la cultura solidaria colectiva, agrícola y artesanal, en cultura de consumo individualista, estimulada por la agroindustria de manera creciente en las últimas décadas. La agroindustria, en gran desarrollo en esta región (producción vitivinícola, ruta del vino, enoturismo), ofrece empleos temporeros precarios, al mismo tiempo que posibilita crédito de consumo local a hombres y mujeres de estos 
grupos vulnerables, deuda que los amarra al sistema con frágiles negociaciones salariales. Las personas, tanto hombres y mujeres que quedan al margen de la contratación para las cosechas y vendimias se dedican a trabajos incluso más precarios, como aquel de "rebusca" de rastrojos, que consiste en recoger los restos de la cosecha para su propio consumo y de frutas silvestres para la venta.

En los propósitos de las entrevistadas apareció la nostalgia por el cultivo familiar e incluso comunitario, el huerto que se cuidaba en épocas de la generación anterior, relacionando el movimiento sísmico con el abandono de tradiciones agrícolas y el alejamiento de la tierra. Resultó sorprendente constatar que la mayoría de las personas tuvieron problemas de sueño y apetito luego del terremoto, pasando días sin probar alimento, lo cual suscita variadas interpretaciones psicológicas, tales como una baja energía psíquica que abandona la catexis de los alimentos, posiblemente relacionada con la creencia de un vínculo entre el abandono del trabajo de la tierra y el suceso catastrófico. Subyacía en estas creencias un mecanismo proyectivo sobre el movimiento sísmico, de los movimientos psicosociales tan radicales sufridos por la comunidad en las últimas décadas en tanto cambio cultural y económico.

Luego de terminadas las entrevistas y el diagnóstico, se convocó a las mismas 52 personas a integrar Grupos Operativos, -grupos de solo hombres coordinado por una dupla de psicólogos varones y grupos de solo mujeres, coordinado por una dupla de psicólogas mujeres ${ }^{7}$, con el objetivo de elaborar los efectos de la situación catastrófica: se organizaron 4 grupos, 2 de mujeres (13 y 18 respectivamente, incluyendo el segundo grupo una mayoría de las asistentes al primero) y 2 de hombres (3 y 4 respectivamente) que se sucedieron en el tiempo con un intervalo de tres semanas.

En el trabajo grupal pudimos observar cómo, a partir de una primera sesión de evaluación de las relaciones de la comunidad, se pudo ir reconstituyendo en el tiempo el tejido social y avanzando en subjetividad política. El grupo de mujeres vivió un proceso notable de socialización grupal informal, entre el primer Grupo Operativo y el segundo, que evidenció una alta capacidad de resiliencia y un buen pronóstico en cuanto a la reconstitución de una subjetividad política que se había debilitado, no solo por la catástrofe, sino por las precarias condiciones colectivas del último tiempo. La diferencia del material que emergió en la primera

7. Según metodología en psicología grupal de género de Teresa Bernárdez y Bárbara Cohn, perteneciente esta última a la International Association of Group Psychotherapy. La base teórica de la separación entre hombres y mujeres se relaciona con la reconstitución narcisística de género y la posibilidad de la multiplicidad de roles que en un grupo mixto se limitan por lo general a las posiciones clásicas de género por la interacción convencional que se instala al interior del grupo. 
sesión grupal en comparación con aquel de la segunda, permitió comprobar que el espacio de Grupo Operativo es un lugar de elaboración y reconstitución luego de situaciones catastróficas y que puede servir de modelo de colectivización que persiste en lo cotidiano informal.

Los niveles implícitos y latentes del discurso grupal de la primera sesión -tanto en el grupo de hombres como en el de mujeres- se caracterizaron por una carencia de energía afectiva colectiva, por la falta de cooperación y comunicación entre los integrantes, y con referentes limitados al grupo familiar. Cada integrante se vinculaba de manera individual "en público" con los/las psicólogos/as, y el recorrido grupal de la resonancia y consonancia, si no era aludido por el/la coordinador/a, no emergía espontáneamente.

En el caso de las mujeres, la experiencia grupal fue reconocida como una "muestra" de la experiencia comunitaria de incomunicación, y resultó en la emergencia de un sentimiento de soledad y culpa por la falta de cooperación vecinal. Se acompañó de una tímida nostalgia frente a lo que en algún momento fueron unos "grupos de madres" que se reunían con alguna periodicidad. En los hombres, se observó un sentido de pérdida de la ilusión colectiva con sus pares, que se reflejó en el ritmo y la calidad de la dinámica grupal, en el tono depresivo y desesperanzado de la sesión.

Durante la primera sesión las mujeres evocaron figuras familiares cercanas y la experiencia a contribuir a la cohesión del grupo familiar, especialmente en momentos de emergencia, era representada como satisfactoria, como si las acciones de sobrevivencia cotidiana más difíciles que las habituales, las transformara en sujetos activas de deseo y proyecto. Se observó un alto nivel de pertenencia al género y clase en un principio de la sesión de modo acrítico. En cuanto al género, los propósitos dan cuenta de la instalación de estereotipos tradicionales y convencionales, especialmente con respecto al rol de responsable del hogar y al comportamiento sexual frente a los compañeros o maridos. Hacia el final de la sesión emergió la queja (en un principio latente e inconsciente) acerca de los mandatos de género que solo les permiten sentirse sujetos de deseo y de acción en función del cuidado de la familia.

Finalmente, las mujeres hacen el insight de reconocer que en su rol y tarea de género no encuentran reciprocidad por parte del grupo familiar, ya que son ellas, a pesar de tener un compañero, que se hacen cargo de toda la tarea doméstica y de toda la responsabilidad respecto de los hijos. Este recorrido, que parte de la satisfacción de cumplir con su tarea de cuidado, hasta la toma de conciencia de un cierto grado de explotación por parte de los más cercanos, se asocia con la culpa por la incomunicación con los/las vecinos/as y con la nostalgia por un colectivo de pares de género. En este punto la dinámica sufre un quiebre, ya que lo que en un 
comienzo de la sesión era reconocido como positivo (ser quienes se hacen cargo de todo el grupo familiar), al final de la misma aparece como limitante de relaciones más variadas, flexibles y complejas. Esto desconcierta y hace circular ansiedades, produciéndose una empatía de género entre las participantes por consonancia de la queja contra el sexismo familiar. Este giro mostró un índice procesual en cuanto a la pertenencia y aprendizaje grupal, lo que podía ser un germen de mejor pronóstico que lo que aparecía por el momento.

Los varones, por su parte, expresaron su indefensión, su frustración e impotencia frente a las catástrofes naturales, viéndose sobrepasados incluso por su propia resistencia psicológica a hacerse cargo de sus emociones. Por momentos y cogidos por sorpresa se quebraron y lamentaron la pérdida de antiguas tradiciones de trabajos comunitarios en las cosechas particulares de algún vecino, como "la trilla a yegua suelta" que proveía anualmente varias ocasiones para juntarse en comunidad y que ciertamente sirvieron de cohesión con su grupo de pares. Se podía leer en los niveles implícito y latente la fatalidad y el escepticismo que se referían a algo más que a la catástrofe inmediata: a las actuales condiciones de vida.

Nuestra evaluación de las primeras sesiones de los Grupos Operativos acusó un pronóstico reservado. Consideramos que la pasividad observada en los/las participantes era síntoma de las tres ansiedades exacerbadas que crean un fondo confuso/ paranoide/depresivo empobrecedor de la subjetividad política. Si bien el discurso consciente -en especial de las mujeres- manifestaba la expectativa de la ayuda por parte de las instituciones, lo que se condice con su situación dependiente de género y clase, ciertos signos latentes apuntaban a la perplejidad, escepticismo y al profundo insight de una indefensión que solo encuentra sustento en otras mujeres de la familia, en las mayores. Se aprecia por momentos deseos de empatizar con los/ as vecinos, reprimidos por las convenciones sociales y la desconfianza: los mayores obstáculos que se aprecian son del orden de la censura social, de malentendidos y rumores, lo que apunta al aislamiento y la privacidad. Esto aparece compensado por una alta pertenencia simbólica a la comuna y a la región.

En resumen, en esta primera sesión estimamos que para los propósitos de reconstrucción del tejido social y la subjetividad política, el bajo nivel de comunicación y cooperación colectiva y la ausencia de empatía, no eran las mejores. La pertenencia a la región ayuda a la raigambre rural, sin embargo no se anticipó -en ese momento- posibilidad de cambio en este grupo. Nos pareció que el proceso de aprendizaje para una posible reconstrucción subjetiva era débil, mostrando una actitud de marginalidad pasiva post traumática que les dificultaba retomar un proyecto básico de supervivencia.

Sin embargo, en la segunda sesión que programamos con los/as mismos/as participantes y abriendo el grupo de mujeres de 13 a 18, pudimos constatar que 
el tiempo transcurrido entre sesión y sesión se había constituido en un proceso que se pusiera en marcha en el primer encuentro grupal. Este proceso de avance en la cohesión del tejido social y en la recuperación de la subjetividad política fue especialmente notable en el grupo de mujeres, que será nuestro referente en esta última parte del artículo. La mejoría de la calidad del discurso y del material latente y manifiesto que pudimos recabar en la segunda sesión del grupo de mujeres, nos prueba que la teoría que sostiene Bernárdez y Cohn del restablecimiento narcisista en grupo de solo mujeres, es efectivo.

En esta segunda sesión grupal, después de un intervalo de tres semanas como ya lo precisamos, acudieron 10 mujeres que habían concurrido al primer encuentro, de manera que fue para ellas su segunda sesión grupal. Este subgrupo se constituyó en el núcleo de liderazgo de progreso grupal frente a "las nuevas integrantes", para la consecución de la tarea, que, esta vez, se logró finalizar con el esbozo de un proyecto colectivo. Las mujeres de la tercera edad son quienes denunciaron la pérdida de la cultura solidaria y generaron en las más jóvenes una reacción de transferencia de la energía afectiva, habitualmente encerrada en el grupo familiar cercano, hacia una representación de vínculos comunitarios abiertos y colectivizados por mediación del vínculo con las mayores restablecido en el aquí y ahora grupal.

La pertenencia al género y a la genealogía de las mujeres de la comuna parece haberse fortalecido entre la primera sesión y la segunda, y podemos observar una fuerte cohesión y empatía en torno al tema de la vejez que, unido a la precariedad laboral y de salud, produce gran ansiedad paranoide y depresiva. Esto relacionado con las quejas sobre la dependencia de los mandatos de género de la sesión anterior -subjetividad activa solo centrada en el grupo familiar-genera un desplazamiento de la ética de cuidado a representaciones colectivas de cooperación y colaboración grupal entre mujeres jóvenes en ayuda a las mayores.

En lugar de los niveles de pasividad de la primera sesión, en esta segunda aparece una marginalidad activa, que tiene un sesgo de excentricidad ${ }^{8}$ al desmarcarse como punto de fuga de la representación de un "grupo de madres" y plantearse un "grupo de amigas de diversas edades". Los mandatos de género se encuentran explícitamente cuestionados en esta sesión y el imaginario social refiere a que en la gran ciudad estos mandatos serían menos rígidos.

En resumen, lo más notable de esta segunda sesión es la capacidad de representarse una red de mujeres, un proyecto empático para mujeres en situación precaria y una mayor comunicación a nivel comunitario.

8. Errázuriz, P., Op. Cit. Indicadores de Género a partir de concepto de bell hooks y Teresa de Lauretis. 
Observamos, finalmente, que un inicio de reconstrucción del tejido social se estaba llevando a cabo, luego de que en la primera sesión se hubiera -de alguna manera- convocado a la aldea interna que sirvió de soporte narcisista, en especial en el caso de las mujeres. La presencia de las mujeres mayores, que no eran parientes de las más jóvenes, propició un desplazamiento de los movimientos vinculares -que efectivamente se dieron luego del terremoto de agrupación en torno a las abuelas- hacia el colectivo comunitario. La nueva vinculación grupal tendió una suerte de puente entre lo que se hallaba enquistado endogámicamente a través de la transferencia hacia las mujeres mayores no emparentadas, pudiendo construir una representación de colectivo de género exogámico.

Para concluir, diremos que la utilización de la técnica de Grupo Operativo con perspectiva de género, esto es la inclusión de las gimnasias mencionadas que fortalecen la representación de la genealogía de las mujeres, de la pertenencia a los colectivos del género, de la semejanza y diferencia con sus pares, de las expectativas como sujeto de deseo y su cumplimiento o su frustración, entre otros, recurre a la circulación del material inconsciente en una suerte de salón de espejos que convoca resonancias y consonancias que refuerzan y/o recrean el narcisismo colectivo de género necesario para el desarrollo de la subjetividad política. Este se hace, entonces, indispensable como intervención comunitaria en momentos de catástrofe.

En un Grupo Operativo de solo mujeres con un equipo de coordinación/observación del mismo sexo, los instrumentos utilizados por las psicólogas que apuntan a la multiplicación representacional haciendo consciente y presente el preconsciente, convocan consonancias, disonancias, semejanzas y diferencias desde el eco de la interpretación psicoanalítica grupal sugerida, a veces solo esbozada en un gesto o en una muletilla ad hoc. La ventaja del uso de la técnica operativa consiste en el develamiento de los materiales reprimidos que, en la matriz grupal, emergen por efecto -precisamente- de la multiplicación y resonancia entres las integrantes, y que la coordinadora, por su forma de operar con el material latente, permite dicha emergencia reconociéndola y señalándola. En este punto, la asimetría entre coordinadoras y coordinadas presenta la ventaja de que el material reconocido tanto por la coordinación, devuelta al grupo por la observación, se vuelve legítimo y manejable para las sujetos. Los fantasmas con los que se enfrentan en el aislamiento de los trabajos o de los quehaceres familiares, se quiebran y adquieren una realidad tangible cuando se reconocen en la colectividad como propios del género y de sus mandatos. Adquieren, entonces, una familiaridad como parte de un imaginario colectivo, refrendados por la interpretación psicoanalítica, lo que permite el desarrollo de una flexibilidad dialógica en el mundo interno en lugar de una inacción rígida y amenazada. Esta legitimación que se adquiere en el trabajo grupal mencionado se traspasa a la acción externa, social, colectiva, si ocurre un 
proceso durante algunas sesiones extendidas en el tiempo. A nuestro parecer, y por eso planteamos una intervención al menos de dos sesiones con un espacio de separación entre ellas, en situaciones límites como son las catastróficas, el proceso se consolida con mayor rapidez en un ritmo de urgencia.

"Hablamos de vínculos internos y de vínculos externos integrados en un proceso de espiral dialéctica" señala Pichón Rivière". Añadimos que esta espiral dialéctica integrada con la genealogía del género en la representación y evocación, así como su desplazamiento a los pares que interlocutan en una sesión, constituye la riqueza para la construcción de la subjetividad política que se genera en el espacio grupal.

\section{REFERENCIAS BIBLIOGRÁFICAS}

Errázuriz, P. Psicología Social y Género: espacio a salvo para mujeres. Santiago de Chile, Ed. De la Elipse, 2006.

Pampliega de Quiroga, A. Proceso de la constitución del mundo interno. Buenos Aires, Ed. Cinco, 1992.

Pichón Rivière, E. Teoría del vínculo. Buenos Aires, Nueva Visión, 1980.

9. Pichón Rivière, E. Teoría del vínculo. Buenos Aires, Nueva Visión, 1980, p.55. 